\title{
IMAGE FUSION USING STEERABLE DYADIC WAVELET TRANSFORM
}

\author{
Iztok Koren ${ }^{\dagger}$, Andrew Laine $e^{\ddagger}$, and Fred Taylor ${ }^{\dagger}$ \\ $\dagger$ Department of Electrical and Computer Engineering \\ $\ddagger$ Computer and Information Science and Engineering Department \\ University of Florida, Gainesville, FL 32611
}

\begin{abstract}
An image fusion algorithm based on multiscale analysis along arbitrary orientations is presented. After a steerable dyadic wavelet transform decomposition of multi-sensor images is carried out, the maximum local oriented energy is determined at each level of scale and spatial position. Maximum local oriented energy and local dominant orientation are used to combine transform coefficients obtained from the analysis of each input image. Reconstruction is accomplished from the modified coefficients, resulting in a fused image. Examples of multi-sensor fusion and fusion using different settings of a single sensor are demonstrated.
\end{abstract}

\section{INTRODUCTION}

Image fusion combines different aspects of information from the same imaging modality or from distinct imaging modalities [1] and can be used to improve the reliability of a particular computational vision task or to provide a human observer with a deeper insight about the nature of observed data.

The simplest method of fusing images is accomplished by computing their average. Features from each original image are present in a fused image, however, the contrast of the original features can be significantly reduced. More sophisicated techniques rely on multiscale representations, such as pyramids [12, 2] and wavelet analysis $[10,6]$ : transform coefficients are fused rather than spatial image pixels, and reconstruction from fused transform coefficients is then computed.

We present a method which executes low-level fusion on registered images by the use of a steerable dyadic wavelet transform. A steerable dyadic wavelet transform incorporates analysis along an arbitrary orientation into a multiscale framework. Steerable filters in the filter bank implementation of a steerable dyadic wavelet transform are employed in quadrature pairs, so that the fusion is based on local oriented energy. It has been shown that the human visual system detects features at the points where local energy, defined as the sum of the squared responses of a quadrature pair of odd-symmetric and even-symmetric filters, is maximum [8]. Local energy reaches its maximum at both lines and edges. It has also been shown that peaks of local energy accurately localize composite edges which are more common in real world images than ideal step edges, whereas linear filters exhibit localization errors [9]. Since features can occur along any orientation we use the local oriented energy to fuse perceptually significant features in corresponding locations of previously registered images across different scales.

This paper is organized as follows. Section 2 formulates a steerable dyadic wavelet transform. Next, the image fusion algorithm is described in Section 3. Results are then shown in Section 4. Finally, Section 5 presents a brief summary.

\section{A STEERABLE DYADIC WAVELET TRANSFORM}

Steerable filters are filters whose arbitrary rotation can be synthesized from a linear combination of basis filters. The filter is steerable if it has a finite number of terms in its Fourier series expansion of its polar angle $[3]$.

A steerable dyadic wavelet transform [5] combines the properties of a discrete dyadic wavelet transform [7] with the analysis along arbitrary orientations. The transform is implemented as a filter bank consisting of polar separable filters. Similar to a steerable pyramid described in [11] the radial portion of the filter bank is designed first and any desired angular variation applied later.

The radial part of the filter bank was derived from the filter bank implementation of a one-dimensional discrete dyadic wavelet transform [7] with the family of filters proposed in [4]:

$$
\begin{aligned}
& H(\omega)=\left(\cos \left(\frac{\omega}{2}\right)\right)^{2 m}, \\
& G(\omega)=-16\left(\sin \left(\frac{\omega}{2}\right)\right)^{2},
\end{aligned}
$$




$$
K(\omega)=-\frac{1}{16} \sum_{l=0}^{2 m-1}\left(\cos \left(\frac{\omega}{2}\right)\right)^{2 l}
$$

where $H(\omega), G(\omega)$, and $K(\omega)$ are digital filter frequency responses and $m \in N$.

The frequency responses are related by

$$
|H(\omega)|^{2}+G(\omega) K(\omega)=1
$$

Figure 1 shows a filter bank implementation of a one-dimensional discrete dyadic wavelet transform using the family of filters from Equation 1.

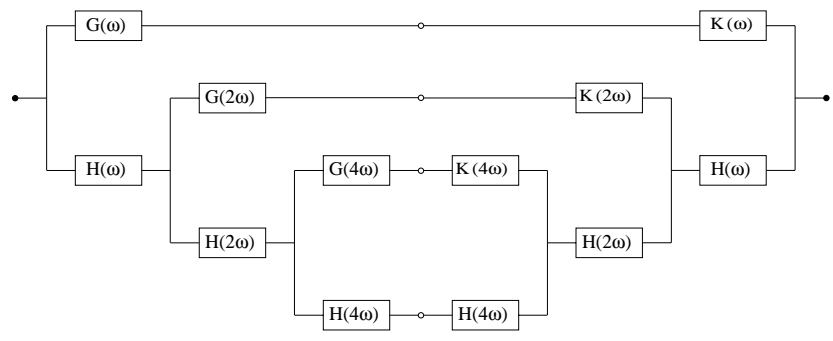

Figure 1: Filter bank implementation of a one-dimensional discrete dyadic wavelet transform decomposition (left) and reconstruction (right) for three levels of analysis.

The wavelets associated with the filter bank using filters from Equation 1 are equal to the second derivative of a spline of degree $2 m+1$, whose Fourier transform is equal to $\left(\frac{4}{\omega} \sin \left(\frac{\omega}{4}\right)\right)^{2 m+2}$.

Let $\omega_{r}=\sqrt{\omega_{x}^{2}+\omega_{y}^{2}}, \omega_{\phi}=\arg \left(\omega_{x}, \omega_{y}\right)$, and let $F\left(\omega_{r}, \omega_{\phi}\right)=F_{R}\left(\omega_{r}\right) F_{\Phi}\left(\omega_{\phi}\right)$ be a filter in the filter bank implementation of a steerable dyadic wavelet transform. The radial part of the filter bank implementation of a steerable dyadic wavelet transform is then comprised of filters $H_{R}\left(\omega_{x}, \omega_{y}\right), G_{R}\left(\omega_{x}, \omega_{y}\right)$, and $K_{R}\left(\omega_{x}\right.$, $\omega_{y}$ ). The filters' frequency responses satisfy

$$
F_{R}\left(\omega_{x}, \omega_{y}\right)= \begin{cases}F\left(\omega_{r}\right) & \text { if } \omega_{r}<\pi \\ F(\pi) & \text { otherwise }\end{cases}
$$

where $F_{R}\left(\omega_{x}, \omega_{y}\right)$ is equal to $H_{R}\left(\omega_{x}, \omega_{y}\right), G_{R}\left(\omega_{x}, \omega_{y}\right)$, or $K_{R}\left(\omega_{x}, \omega_{y}\right)$, and $F(\omega)$ is one of the filters defined previously in Equation 1.

The angular portion of the frequency responses of filters in the filter bank is constant for all filters except $G\left(\omega_{x}, \omega_{y}\right)$. The frequency response $G_{\Phi}\left(\omega_{x}, \omega_{y}\right)$ was chosen to be

$$
G_{\Phi}\left(\omega_{x}, \omega_{y}\right)=\cos ^{2 n}\left(\omega_{\phi}\right),
$$

where $n \in \boldsymbol{N}$.

This frequency response can be steered with $2 n+1$ basis filters (the minimum number of basis filters required to steer a steerable filter is equal to the number of nonzero coefficients in a Fourier series expansion of the filter along its polar angle [3]).

The filters in the filter bank implementation of the steerable dyadic wavelet transform were therefore

$$
\begin{aligned}
H\left(2^{p} \omega_{x}, 2^{p} \omega_{y}\right) & =H_{R}\left(2^{p} \omega_{x}, 2^{p} \omega_{y}\right), \\
G_{k}\left(2^{p} \omega_{x}, 2^{p} \omega_{y}\right) & =G_{R}\left(2^{p} \omega_{x}, 2^{p} \omega_{y}\right) G_{\Phi}^{\theta_{k}}\left(\omega_{x}, \omega_{y}\right), \text { and } \\
K\left(2^{p} \omega_{x}, 2^{p} \omega_{y}\right) & =K_{R}\left(2^{p} \omega_{x}, 2^{p} \omega_{y}\right),
\end{aligned}
$$

where $G_{\Phi}^{\theta_{k}}\left(\omega_{x}, \omega_{y}\right)$ denotes $G_{\Phi}\left(\omega_{x}, \omega_{y}\right)$ rotated by $\theta_{k}$, $\left\{\theta_{k}\right\}=\left\{\frac{k}{2 n+1} \pi\right\}, k \in\{0,1,2, \ldots, 2 n\}$, and level $(p+1) \in$ $N$.

The interpolation functions needed to steer $G_{k}\left(\omega_{x}\right.$, $\left.\omega_{y}\right)$ were $a_{k}(\theta)=\frac{1}{2 n+1}\left(1+2 \sum_{l=1}^{n} \cos \left(2 l\left(\theta-k \Delta \theta_{k}\right)\right)\right)$, where $\Delta \theta_{k}=\frac{\pi}{2 n+1}$. Arbitrary rotation of $G_{\Phi}\left(\omega_{x}, \omega_{y}\right)$ can then be expressed as

$$
G_{\Phi}^{\theta}\left(\omega_{x}, \omega_{y}\right)=\sum_{k=0}^{2 n} a_{k}(\theta) G_{\Phi}^{\theta_{k}}\left(\omega_{x}, \omega_{y}\right) .
$$

Figure 2 shows the magnitude frequency responses of filters $H\left(\omega_{x}, \omega_{y}\right), K\left(\omega_{x}, \omega_{y}\right)$, and $G_{k}\left(\omega_{x}, \omega_{y}\right)$ with $n=1$ in Equations 2 and 3.

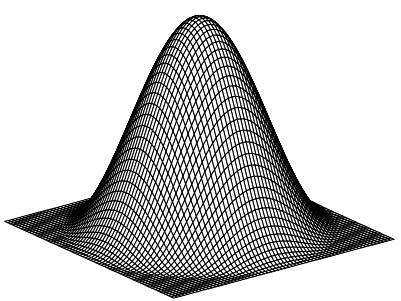

(a)

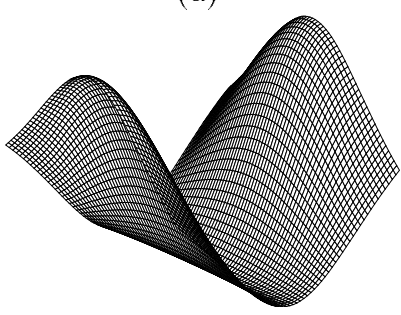

(c)

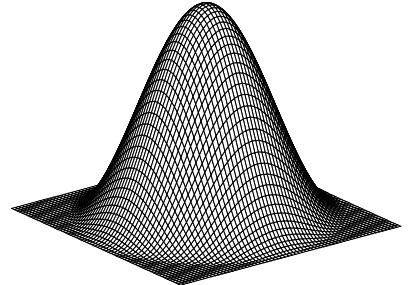

(b)

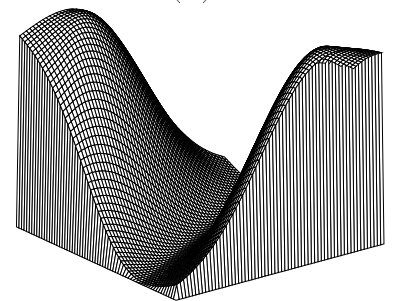

(d)

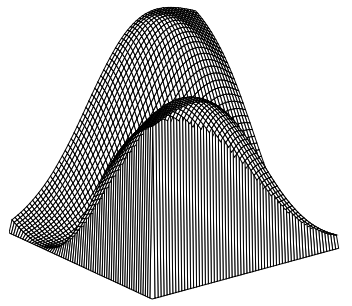

(e)

Figure 2: The magnitude frequency responses of: (a) Filter $H\left(\omega_{x}, \omega_{y}\right)$. (b) Filter $K\left(\omega_{x}, \omega_{y}\right)$. (c)-(e) Filters $G_{k}\left(\omega_{x}, \omega_{y}\right)$.

A steerable dyadic wavelet transform decomposition can be viewed as circularly symmetric smoothing 
at different scales, followed by an arbitrarily steered oriented second derivative.

Reconstruction from outputs of filters $G_{\Phi}^{\theta_{k}}\left(\omega_{x}, \omega_{y}\right)$ is based upon the fact that the sum of $\theta_{k}$ rotated filters $G_{\Phi}\left(\omega_{x}, \omega_{y}\right)$ is equal to a constant $C_{2 n}$ :

$$
\sum_{l=0}^{2 n} \cos ^{2 n}\left(\omega_{\phi}-\frac{l}{2 n+1} \pi\right)=C_{2 n} .
$$

\section{IMAGE FUSION}

For image fusion, steerable filters from the filter bank implementation of a steerable dyadic wavelet transform were used in quadrature pairs (i.e., with their Hilbert transform counterparts). The Hilbert transform of filter frequency responses $G_{\Phi}\left(\omega_{x}, \omega_{y}\right)$ (Equation 2) is

$$
G_{\Phi_{H}}\left(\omega_{x}, \omega_{y}\right)=-j \operatorname{sgn}\left(\cos \left(\omega_{\phi}\right)\right) \cos ^{2 n}\left(\omega_{\phi}\right),
$$

where $n$ is the same as in Equation 2 and

$$
\operatorname{sgn}(x)= \begin{cases}1 & \text { if } x \geq 0 \\ -1 & \text { if } x<0\end{cases}
$$

Filters $G_{\Phi_{H}}\left(\omega_{x}, \omega_{y}\right)$ are not steerable. They were approximated with truncated Fourier series expansion by saving only a few maximum magnitude coefficients. Basis filters needed to steer an approximation to the filter frequency response $G_{\Phi_{H}}\left(\omega_{x}, \omega_{y}\right)$ were then added to the filter bank implementing a steerable wavelet transform, so that the output of filters $G_{R}\left(2^{p} \omega_{x}, 2^{p} \omega_{y}\right)$ were not multiplied by $G_{\Phi}^{\theta}\left(\omega_{x}, \omega_{y}\right)$ alone, but also with approximation to $G_{\Phi_{H}}^{\theta}\left(\omega_{x}, \omega_{y}\right)$, where $\theta$ denotes some arbitrary rotation. Thus, quadrature pairs of filters steered to some arbitrary angle $\theta$ were used to determine the local oriented energy, which was defined as the sum of the squared output from each filter of the quadrature pair.

For images to be fused, a steerable dyadic wavelet transform was first carried out. Next, local oriented energy obtained from the quadrature pair of steerable filters was computed and the local dominant orientation (i.e., the angle that maximized the local oriented energy) was determined at each level and position [3]. Filters were then steered to the local dominant orientation, and local oriented energies were compared. The coefficients corresponding to the greater local oriented energy were included for reconstruction. The final reconstruction was accomplished with filters $G_{\Phi}\left(\omega_{x}, \omega_{y}\right)$ (Equation 4), while filters $G_{\Phi_{H}}\left(\omega_{x}, \omega_{y}\right)$ were used only for computation of local oriented energy.

\section{RESULTS}

For the results presented in this section our fusion algorithm was executed with $m=1$ in Equation 1 and $n=1$ in Equations 2, 3, and 5 (the sum of rotated filters $G_{\Phi}\left(\omega_{x}, \omega_{y}\right)$ in Equation 4 was equal to $\left.C_{2}=1.5\right)$.

A simple example of image fusion for extending the depth of focus of a camera is demonstrated in Figure 3 . A pair of images with distinct areas in focus was first fused manually (cut and paste), and then by our fusion algorithm. As in [6] we compared the ideal (manually fused) result with the output of our algorithm. The mean-square error (MSE) for the output of our algorithm was 12.88 and the MSE when the fused image was simply the average of the two images was 113.51 .

A sample of multisensor data is shown next. Figure 4 shows Channels 1 and 5 of Landsat TM images of Sunbury, and the image fused with our algorithm.

\section{CONCLUSION}

The described algorithm performed image fusion across multiple scales and along arbitrary orientations. Overcompleteness of a steerable dyadic wavelet transform demonstrated advantages: the transform was shift-invariant and there allowed no aliasing in the filter bank implementation, both properties are highly desirable for image fusion applications.

Steerable filters in the filter bank implementation of a steerable dyadic wavelet transform were designed in quadrature pairs to compute the maximum local oriented energy and the local dominant orientation at each level and position. In addition to perceptual significance of the maximum local energy, computing the maximum local oriented energy introduced no localization error and enabled comparison of corresponding features within distinct input images.

\section{ACKNOWLEDGMENT}

The authors wish to thank Dr. Bruce Chapman of the Jet Propulsion Laboratory, Pasadena, CA, for kindly providing the multi-sensor images. This work was supported in part by National Science Foundation grant No. IRI-9111375.

\section{REFERENCES}

[1] J. K. Aggarwal, Multisensor Fusion for Computer Vision, Berlin Heidelberg: Springer-Verlag, 1993.

[2] P. J. Burt and R. J. Kolczynski, "Enhaced image capture through fusion," in Proc. 4th Int. Conf. Comput. Vision, Berlin, Germany, May 1993, pp. $173-182$.

[3] W. T. Freeman and E. H. Adelson, "The design and use of steerable filters," IEEE Trans. Pattern Anal. Machine Intell., vol. PAMI-13, pp. 891-906, 1991. 


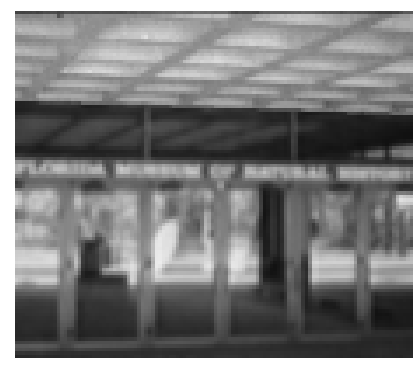

(a)

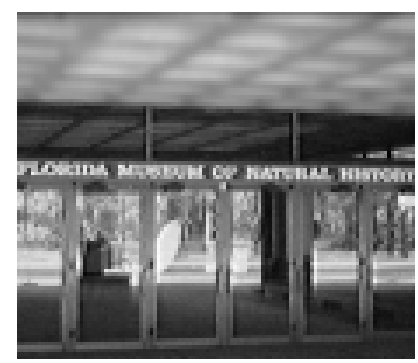

(b)

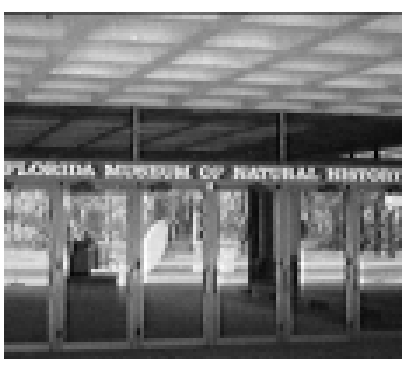

(c)

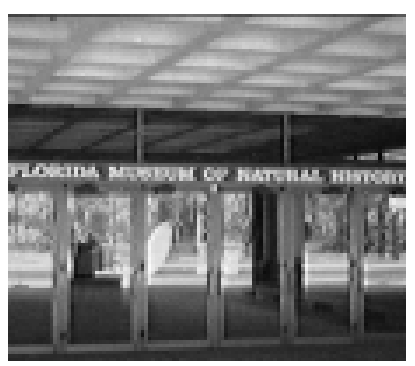

(d)

Figure 3: (a) An image with lower part blurred. (b) An image with upper part blurred. (c) Fused image obtained by combining images from (a) and (b) manually. (d) Fused image resulting from our fusion algorithm.

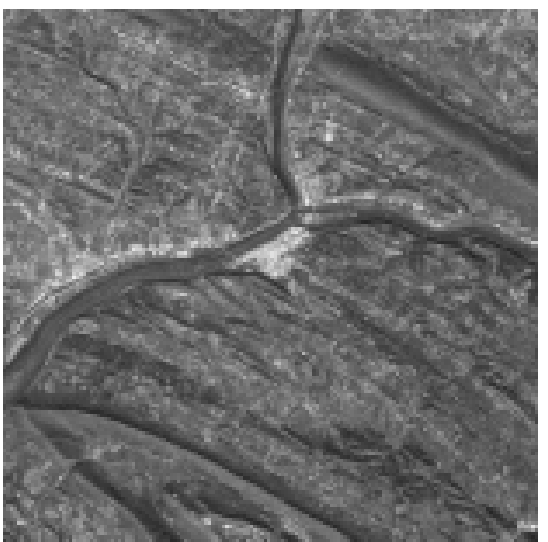

(a)

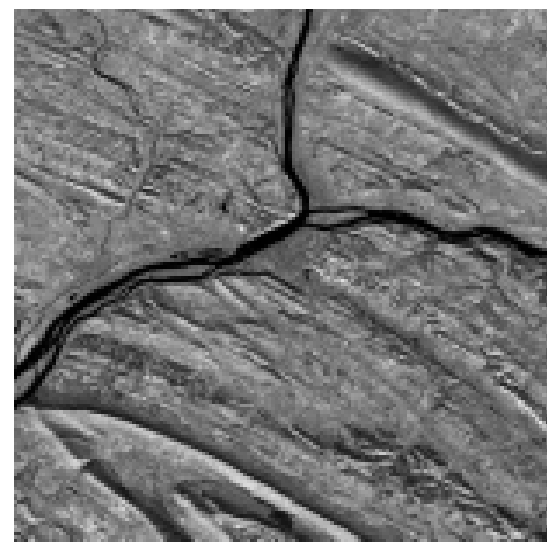

(b)

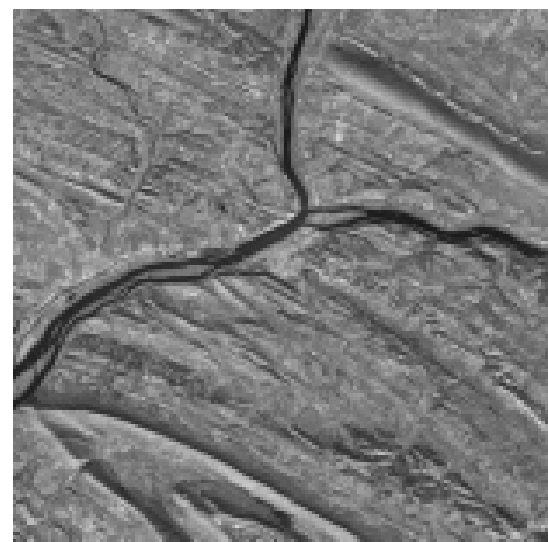

(c)

Figure 4: (a) TM-1 image. (b) TM-5 image. (c) Fused image using our fusion algorithm.

[4] A. Laine, J. Fan, and S. Schuler, "A framework for contrast enhancement by dyadic wavelet analysis," in Digital Mammography, A. G. Gale, S. M. Astley, D. R. Dance, and A. Y. Cairns, Eds., Amsterdam, The Netherlands: Elsevier, 1994, pp. 91-100.

[5] A. Laine, I. Koren, W. Yang, and F. Taylor, "A steerable dyadic wavelet transform and interval wavelets for enhancement of digital mammography," in Wavelet Applications II, H. H. Szu, Ed., Proc. SPIE, Orlando, FL, Apr. 1995, vol. 2491, pp. 736-749.

[6] H. Li, B. S. Manjunath, and S. K. Mitra, "Multisensor image fusion using the wavelet transform," in Proc. IEEE Int. Conf. Image Processing, Austin, TX, Nov. 1994, vol. 1, pp. 51-55.

[7] S. Mallat and S. Zhong, "Characterization of signals from multiscale edges," IEEE Trans. Pattern Anal. Machine Intell., vol. PAMI-14, pp. 710-732, 1992.

[8] M. C. Morrone and D. C. Burr, "Feature detection in human vision: a phase-dependent energy model," Proc. Royal Soc. London, vol. 235, pp. 221-245, 1988.

[9] P. Perona and J. Malik, "Detecting and localizing edges composed of steps, peaks and roofs," in Proc. 3rd Int. Conf. Comput. Vision, Osaka, Japan, Dec. 1990, pp. 52-57.

[10] T. Ranchin, L. Wald, and M. Mangolini, "Efficient data fusion using wavelet transform: the case of SPOT satellite images," in Mathematical Imaging: Wavelet Applications in Signal and Image Processing, A. F. Laine, Ed., Proc. SPIE, San Diego, CA, Jul. 1993, vol. 2034, pp. 171-178.

[11] E. P. Simoncelli, W. T. Freeman, E. H. Adelson, and D. J. Heeger, "Shiftable multiscale transforms," IEEE Trans. Inform. Theory, vol. IT-38, pp. 587-607, 1992.

[12] A. Toet, "Image fusion by a ratio of low-pass pyramid," Pattern Recognition Letters, vol. 9, pp. 245253,1989 . 